References:

[1] Riemer H.J.A. Slart et al. FDG-PET/CT(A) imaging in large vessel vasculitis and polymyalgia rheumatica: joint procedural recommendation of the EANM, SNMMI, and the PET Interest Group (PIG), and endorsed by the ASNC. Eur J Nucl Med Mol Imaging. 2018 Jul;45(7):1250-69.

Table. Performance of different threshold for maximal thickness of the aortic wall for diagnosis of aortitis using PET positivity as the gold standard*.

\begin{tabular}{llllll}
\hline Thickness $(\mathrm{mm})$ & Sensitivity & Specificity & NPV & PPV & accuracy \\
\hline$\geq 1.9$ & $100 \%$ & $30.4 \%$ & $100 \%$ & $29.41 \%$ & $46 \%$ \\
$\geq 2.0$ & $100 \%$ & $31.88 \%$ & $100 \%$ & $29.85 \%$ & $47 \%$ \\
$\geq 2.2$ & $95 \%$ & $53.62 \%$ & $97.36 \%$ & $37.25 \%$ & $63 \%$ \\
$\geq 2.6$ & $85 \%$ & $71.01 \%$ & $94.23 \%$ & $45.94 \%$ & $74 \%$ \\
$\geq 3.0$ & $60 \%$ & $84.05 \%$ & $87.88 \%$ & $52.17 \%$ & $78 \%$ \\
$\geq 3.3$ & $60 \%$ & $92.75 \%$ & $88.89 \%$ & $70.59 \%$ & $85 \%$ \\
$\geq 3.5$ & $35 \%$ & $95.65 \%$ & $83.54 \%$ & $70 \%$ & $82 \%$ \\
$\geq 3.8$ & $25 \%$ & $98.55 \%$ & $81.92 \%$ & $83.33 \%$ & $82 \%$ \\
$\geq 4.0$ & $20 \%$ & $100 \%$ & $81.17 \%$ & $100 \%$ & $82 \%$
\end{tabular}

${ }^{*}$ Made with ROC curve in descendant thoracic and suprarenal segment of the aorta; PET: ${ }^{18} \mathrm{~F}$ FDG PET/CT; NPV: negative predictive value; PPV: positive predictive value.

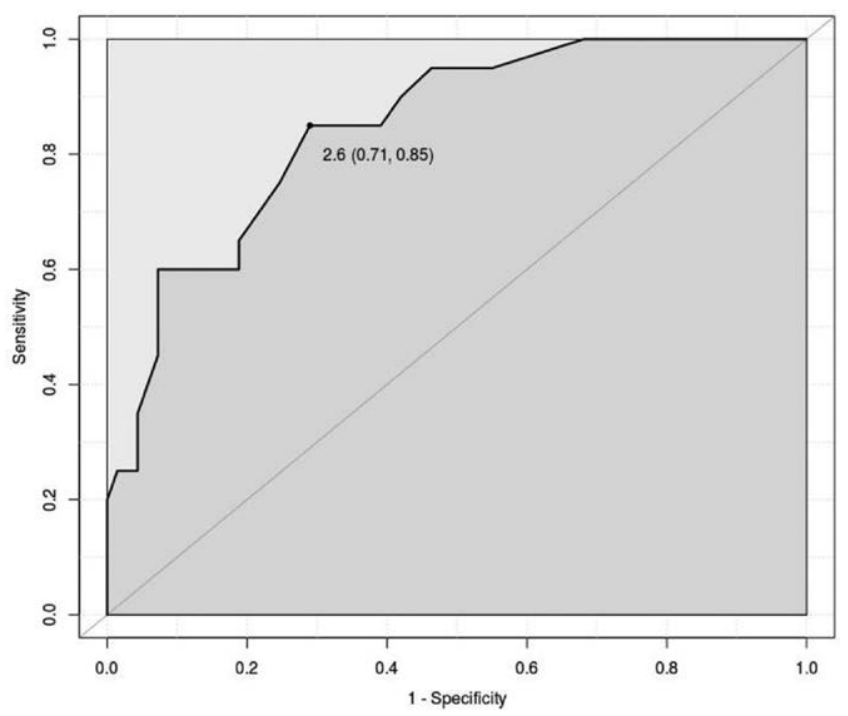

Figure. ROC curve of aortic wall thickness, in descendant thoracic and suprarenal abdominal segments for the diagnosis of aortitis.

Disclosure of Interests: None declared

DOI: 10.1136/annrheumdis-2020-eular.2804

\section{AB1096 AND PATIENT REPORTED OUTCOMES IN SPONDYLOARTHRITIS AND PSORIATIC ARTHRITIS: A SYSTEMATIC LITERATURE REVIEW}

T. Gudu' 1 , M. A. D'agostino ${ }^{1} .{ }^{1}$ Ambroise Pare Hospital, Rheumatology, Boulogne-Billancourt, France

Background: Patient reported outcomes (PROs) reflect patients' opinion on disease activity and impact of disease on various aspects of life. They are essential in the assessment of patients spondyloarthritis (SpA) and psoriatic arthritis (PsA), but may be influenced by several factors other than disease activity and severity. Ultrasound (US) is an objective tool to evaluate inflammation and structural damage in these conditions.

Objectives: The objective of this study was to determine if and to which extent the US assessment reflects patient perspective in patients with SpA and PsA.

Methods: Two separate systematic literature reviews were conducted for each disease. The research questions were formulated according to the PICO framework. The patient reported domains of health were selected from the ones included in the Core Set for SpA [1] and PsA [2] and from the ones frequently reported in clinical trials and observational studies for SpA and PsA, as well as patient self-assessment of pain at the joint level. We
Table 1. Patient reported domains evaluated in the included studies.

\begin{tabular}{llc}
\hline & Domain & Studies reporting the domain \\
& $\mathrm{N}(\%)$ \\
\hline SpA & Disease activity & $11(91.7)$ \\
& Function/ disability & $9(75)$ \\
& Pain & $7(58.3)$ \\
& Patient global assessment & $5(41.7)$ \\
Quality of life & $5(41.7)$ \\
Morning stiffness & $3(25)$ \\
Fatigue & $1(8.3)$ \\
Sleep & $1(8.3)$ \\
Global health & $1(8.3)$ \\
Patient reported peripheral swelling & $1(8.3)$ \\
Function/ disability & $10(83.3)$ \\
Pain & $9(75)$ \\
Patient global assessment & $8(66.7)$ \\
Disease activity & $5(41.7)$ \\
Quality of life & $4(33.3)$ \\
Disease impact & $3(25)$ \\
Mood disorders & $2(16.7)$ \\
Fatigue & $2(16.7)$ \\
Morning stiffness & $1(8.3)$
\end{tabular}

included articles that evaluated any kind of relationship between PROs and US assessment.

Results: $\mathrm{SpA}$

In total, 2977 abstracts were identified, of which 12 articles were retained in the final analysis: 10 cross-sectional studies assessing disease activity state and 2 longitudinal, non-interventional trials assessing treatment response. The most frequently evaluated domains of health are depicted in table 1.

The cross-sectional studies evaluating only one joint area (foot and ankle, sacroiliac joint, shoulder and hip) showed that overall there is no important association between the PROs and the US parameters. In studies evaluating multiple entheses sites there were several correlations between PROs (pain, disease activity, function, quality of life (QoL)) and US variables, i.e., US inflammatory scores and total enthesitis scores, comprising both inflammatory and structural changes $(r=0.24-0.53)$. In the longitudinal studies there was no consistent association between PROs and US variables.

PsA

Out of the 1267 abstracts identified, 12 were finally included in the qualitative analysis: 5 cross-sectional studies on disease activity state and 7 longitudinal studies, of which 2 on disease activity state and 5 on treatment response. The most frequently evaluated domains of health are depicted in table 1

Overall, in the cross-sectional studies, there was no consistent association between PROs and US assessment. In the longitudinal studies on disease activity, pain and patient global assessment (PGA) were associated with US inflammatory variables $(r=0.22-0.28)$, while disability correlated with US damage $(r=0.22-0.39)$. Change in PGA and pain was associated with change in the global US inflammation score $(r=0.28-0.35)$. In longitudinal studies on treatment response results were inconsistent: two studies showed that there was significant parallel trend of some PROs (function, pain and PGA) and some US entheses scores, but the other 3 studies showed no significant association.

Conclusion: The association between PROs and US in SpA and PsA was rather inconsistent. Patient perspective and US examination seem to provide different, but complementary perspectives on disease assessment and both should be taken into account in SpA and PsA management.

References:

[1] van der Heijde, J Rheumatol 1999

[2] Orbai, An Rheum Dis 2016

Acknowledgments: Supported by PARTNER Fellowship

Disclosure of Interests: None declared

DOI: 10.1136/annrheumdis-2020-eular.4316

\section{\begin{tabular}{|l|l}
\hline AB1097 THE ASSOCIATION OF ULTRASOUND ASSESSMENT \\
\hline
\end{tabular} AND PATIENT REPORTED OUTCOMES IN RHEUMATOID ARTHRITIS: A SYSTEMATIC LITERATURE REVIEW}

T. Gudu' ${ }^{1}$, M. A. D'agostino'. ' Ambroise Pare Hospital, Rheumatology, Boulogne-Billancourt, France

Background: Patient reported outcomes (PROs) are recognized to be essential outcome measures in the assessment of patients with rheumatoid arthritis (RA) but they can be prone to being influenced by multiple variables. Thus, objective 